\title{
Design and Application of Ultrasonic Measurement Systems for Akashiwo Sanguinea
}

\author{
Jieli Qin ${ }^{1,2}$, Shaoping Shang ${ }^{2,3 *}$ \\ ${ }^{1}$ Department of Communication Engineering, Xiamen University, Xiamen 361005, China \\ ${ }^{2}$ Key Laboratory of Underwater Acoustic Communication and Marine Information Technology, (Xiamen University) Ministry \\ of Education, Xiamen 361005, China \\ ${ }^{3}$ College of Oceanography and Enviromental Science, Xiamen University, Xiamen 361005, China
}

Corresponding Author Email: iqin_sea@163.com

https://doi.org/10.18280/ts.360112

Received: 18 December 2018

Accepted: 5 February 2019

\section{Keywords:}

ultrasonic measurement, Akashiwo sanguinea (A. sanguinea), acoustic doppler velocimenter $(A D V)$, development board $(D B)$, integrated backscattered strength (IBS), algea cell concentration

\begin{abstract}
The red tide caused by Akashiwo sanguinea (A. sanguinea) has a serious negative impact on the ecological environment. Thus, it is of great importance to monitor the changes of algae cell concentration and thus forecast the red tide. This paper sets up two ulstrasonic systems based on the backscattered sound of algae cells, and uses them to measure the acoustic signals of $A$. sanguinea solutions of 17 different concentrations. The two systems were respectively developed on acoustic Doppler velocimenter (ADV) and single-probe development board (DB). The measured signals were analyzed to disclose the correlation between the integrated backscattered strength (IBS) and the algae cell concentration. The results show that both ADV system and DB system worked effectively when the algae cell concentration fell between $0.1 \times 10^{5}$ cells/L and $0.9 \times 10^{5}$ cells/L; The IBS continued to increase with the growth in algae cell concentration, but the growth rate was dampened when the concentration reached $2 \times 10^{5}$ cells/L, leading to fluctuations in the growing trend; the dampening is the result of the acoustic attenuation effect of the algae cells in medium-high concentration range. The test results of the ultrasonic systems agreed well with the theoretical predictions after particle size correction, indicating that attenuation compensation can enhance the system performance. The proposed systems can realize real-time monitoring of algae cell concentration, laying a solid basis for prewarning of red tide.
\end{abstract}

\section{INTRODUCTION}

Red tide, one of the hazards of marine environment [1-3], will destroy the stability of marine ecosystem, affect human life [4-5], and even cause great loss. It is particularly important to find how to minimize the impact of red tide disasters, or form early warning. Scholars have obtained certain achievements in the prevention, reduction and control of red tide [6-7].

At present, a large number of technologies are applied to the observation and detection of red tide, but the main monitoring means are divided into three categories [8-9]: field sampling, satellite remote sensing and acoustic observation, as shown in Figure 1. Although a small amount of raw liquor can be more accurately classified into algae species in the most traditional field sampling method, it is a single-point measurement and time-consuming and labor-consuming. For the latter two methods that are gradually popular monitoring means, although the classification ability is weak, the space-time resolution ability is better than field sampling. In the acoustic method, the satellite remote senser is not required to carry out wide-scale monitoring, is not affected by the weather, and the processing time is short. The greatest advantage is that it can monitor at the early stage of the occurrence of red tide to achieve the purpose of forecasting, which is of practical significance to prevent the occurrence of red tide.

The development of acoustic technology in marine monitoring is mature and it has been widely used in the study of plankton [10-11], especially the majority of large target zooplankton [12-14]. The biological morphology and physical properties are analyzed by means of acoustic volume scattered. However, the particle size of microalgae is between $1 \mu \mathrm{m}$ and $200 \mu \mathrm{m}$, which puts forward higher requirements for acoustic methods. For skeletonema costatum, Blanc et al. measure the bulk scattered strength in the laboratory by using $2.6 \mathrm{Mhz}$ ultrasound and compare the time domain and frequency domain of pure water and those containing skeletonema costatum. However, a further analysis is not given [15]. Kim et al., South Korean scholars, have carried out a series of studies on the species of red tide algae. They use a $5 \mathrm{Mhz}$ probe to study and analyze the change of acoustic signal characteristics during the diurnal division of algal cells [16]. The $g$ and $h$ values of algae are measured by experiments, and the backscattered strength characteristics of 6 kinds of concentration of algae in the laboratory are studied with $5 \mathrm{Mhz}$ ultrasound [17]. Two kinds of high frequency ultrasound (5Mhz and $10 \mathrm{Mhz}$ ) are used to study the backscattering of alga fluid with specific concentration in laboratory and sea test respectively. $\mathrm{ADV}$, as a $6 \mathrm{Mhz}$ ultrasonic Doppler velocimeter, is usually used in flow velocity measurement and sediment correlation monitoring [19-21]. However, direct microalgae measurement is rarely mentioned. Therefore, it is worthy of further discussion and research whether ADV is suitable for algae concentration monitoring, whether applicability of ultrasonic backscattered detection method to algae cell concentration can be popularized, and whether probe 
frequency can be reduced.

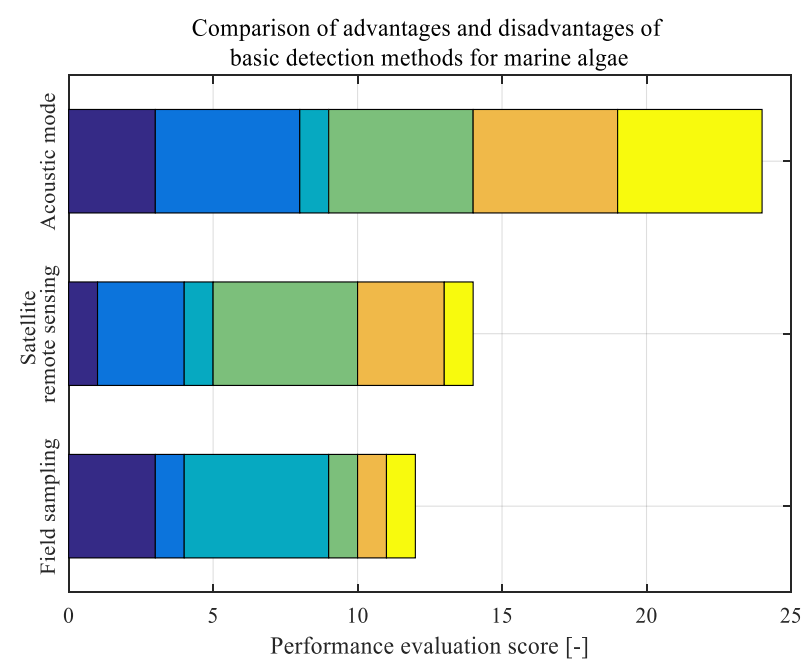

Figure 1. Comparison of advantages and disadvantages of basic methods of marine algae monitoring (red tide)

(1) Three methods: acoustic observation, field sampling and satellite remote sensing; (2) each color block corresponds sampling volume, sampling frequency, algae species identification capability, system space-time resolution accuracy, system processing time and early monitoring of red tide occurrence. Taking the 5-point system as an example, 1 point means general (poor) perfirmance, 3 points mean good performance (moderate applicability), and 5 points mean excellent performance (advantage). The longer the color block is, the more outstanding the ability is.

In this study, the ultrasonic microalgae detection of Akashiwo sanguinea is designed on the background of particle acoustics. Firstly, the basic principle and measurement model of backward acoustic scattering are introduced, and then the experimental preparation and platform construction are described. Then ADV and DB independent systems are used to carry out ultrasonic detection experiment on the multiconcentration Akashiwo sanguinea solution. Finally, analysis, discussion and summary to the experimental result are carried out.

\section{PRINCIPLE OF ACOUSTIC BACKSCATTERED MEASUREMENT}

\subsection{Backward acoustic scattering of particles}

Ultrasound propagates in a medium containing particle. In addition to transmission sound waves, ultrasound waves caused by particle scattering also carry information about scatterers. According to the Rayleigh model [22-23], the scattering behavior of particles essentially depends on the ratio ka of particle size to wave length, and its dimensionless expression is shown in Formula (1), where $d$ is particle diameter, $\mathrm{a}$ is radius, $\mathrm{f}$ is frequency of sound wave, $\mathrm{k}$ is wave number, and $\lambda$ is wave length.

$k a=\frac{2 \pi}{\lambda} \cdot \frac{d}{2}=\frac{\pi \cdot d}{\lambda}=\frac{\pi \cdot f \cdot d}{c}=\frac{2 \pi \cdot f \cdot a}{c}$

The directivity function $\mathrm{R}(\theta)$ of the scattered sound field at the far field distance $r$ after the rigid sphere is incident on the plane wave is as shown in Formula $(2)$, where $P_{1}(\cos \theta)$ is a Legendre function; $\mathrm{j}_{1}(\mathrm{ka})$ is a spherical Bessel function; $\mathrm{h}_{\mathrm{l}}^{2}(\mathrm{ka})$ is the second kind of spherical Hank function.

$$
\begin{aligned}
& R(\theta)=\frac{1}{k a} \sum_{l=0}^{\infty} b_{l} e^{j \frac{l+1}{2} \pi} P_{l}(\cos \theta)=\frac{1}{k a} \sum_{l=0}^{\infty}(-j)^{l}(2 l+ \\
& \text { 1) } \frac{\frac{d\left[j_{l}(k a)\right]}{d(k a)}}{\frac{d\left[h_{l}^{2}(k a)\right]}{d(k a)}} \cdot e^{j \frac{l+1}{2} \pi} P_{l}(\cos \theta)
\end{aligned}
$$

The polar coordinate profile of acoustic scatter at different ka values at angle $\theta$ is shown in Figure 2. The acoustic scattered strength is normalized to define that scattering occurs in the direction of $0^{\circ}$ in accordance with the direction of the incident wave is forward scattered, and scattering occurs in the direction of $180^{\circ}$ is backscattered. When $\mathrm{ka}<1$, the backscattering is more uniform than that of forward scattering. With the increase of ka, both get enhanced, the enhancement rate of backscattering decreases gradually, the strength of scattered sound wave shows obvious non-uniformity of spatial distribution, and the number of petals in the scattered pattern increases. When $\mathrm{ka} \gg 1$, the backscattering is obviously weakened, the forward scattered strength is obvious, and the directivity is enhanced.
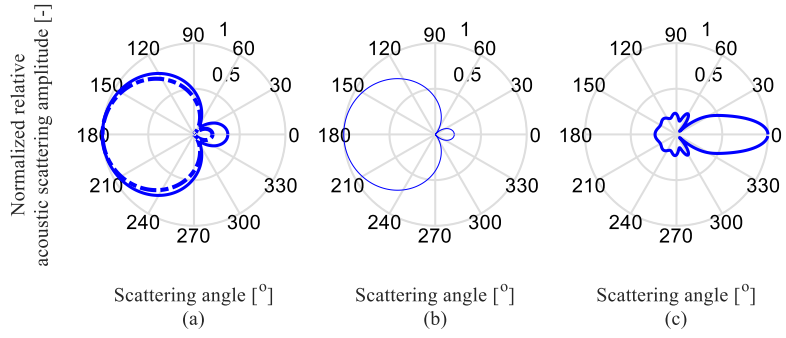

Figure 2. Polar coordinate profile of particle scattering (a) dashed line $\mathrm{ka}=0.09$, full line $\mathrm{ka}=0.9,(\mathrm{~b}) \mathrm{ka}=0.18$, (c) $\mathrm{ka}=6$

In conclusion, for the single-celled planktonic Akashiwo sanguinea with a particle size of about $44 \mu \mathrm{m}$, it is equivalent to an independent sphere with a certain hardness, and the Rayleigh scattered law is also followed. When the incident sound is ultrasound ( $1 \mathrm{Mhz}, \mathrm{ka} \approx 0.09$, as shown by the solid full line of Figurel (a)). Although the absolute value of the backscattered strength of a single particle is weak, it is feasible to collect the backscattered signal since the detection target is an aggregate of a large number of microalgae.

\subsection{Basic application principle}

The ratio of backscattered strength to incident sound wave strength is defined as $\sigma$. When the abundance value of algae cells in seawater is $\mathrm{N}$, the effect of complex scattering is ignored. The corresponding scattered strength $S_{v}$ of the backward acoustic body is obtained as shown in the Formula (3) by directly accumulating the number of particles, and $C_{e}$ is an environmental factor:

$S_{v}=10 \log _{10}(N \sigma)+C_{e}$

$\sigma$ is calculated by using Formula (4) for a fluid pellet present in the solution environment, where, g represents the density ratio of the algal cells to the seawater medium, and $h$ corresponds to the ratio of the two propagating sound velocities.

$\sigma=k^{4} a^{6}\left[\frac{1-g h^{2}}{3 g h^{2}}+\frac{1-g}{1+2 g}\right]^{2}$ 
The algae density can be obtained by centrifugal gradient stratification method [24], and g [25] is obtained after the ratio of algae to seawater. Due to the limitation of experimental equipment, this experiment doesn't measure the actual $g$ and $h$ values [26] of Akashiwo sanguinea. By referring to the existing documents $[27,28,29]$, the following range of values is temporarily limited: $\mathrm{g}$ is $1.04-1.06, \mathrm{~h}$ is $1.02-1.08$. We take $\mathrm{g}=1.05, \mathrm{~h}=1.05$ of Akashiwo sanguinea in this experiment.

It is difficult to measure the backscattered strength directly by experiment. The theoretical value $S_{v}$ has a certain correspondence with the comprehensive characteristic value of IBS, so the acoustic signal data of alga solution can be analyzed. The IBS values of the respective concentrations are compared and calculated to estimate the number of particles. In the same measurement environment and test system, IBS is defined as:

IBS $=20 \log _{10}\left(\frac{\mathrm{V}_{\mathrm{rms}} \mathrm{BS}}{\mathrm{V}_{\mathrm{rms}} \text { Ref }}\right)$

$\mathrm{V}_{\mathrm{rms}} \mathrm{BS}$ is the signal effective voltage value (RMS means root mean square voltages) measured by the seawater algae solution containing Akashiwo sanguinea, and $\mathrm{V}_{\mathrm{rms}}$ Ref is algae-free pure seawater.

\section{EXPERIMENT DESCRIPTION}

\subsection{Culture of Akashiwo sanguinea}

The Akashiwo sanguinea used in the experiment (aseptic strain is provided by the marine ecology laboratory of Xiamen University) is transferred for several times in a special sterile culture room. The seawater is filtered with $0.22 \mu \mathrm{m}$ cellulose acetate filter membrane in the west sea area of Xiamen, sterilized under high pressure and then cooled for storage. Enrich with $f / 2$ nutrient solution [30] (Guillard 1975) rich in various nutrients and vitamins and sterilized, adjust the $\mathrm{pH}$ value of seawater to $8.0 \pm 0.2$. The salinity value is adjusted to $30 \mathrm{psu}(26-34 \%$ ) as a culture medium for algae cells to be propagated and cultured. The culture temperature is $23{ }^{\circ} \mathrm{C}(18-$ $25^{\circ} \mathrm{C}$ ) by alternating between light (3000 lux) and dark cycles every 12 hours.

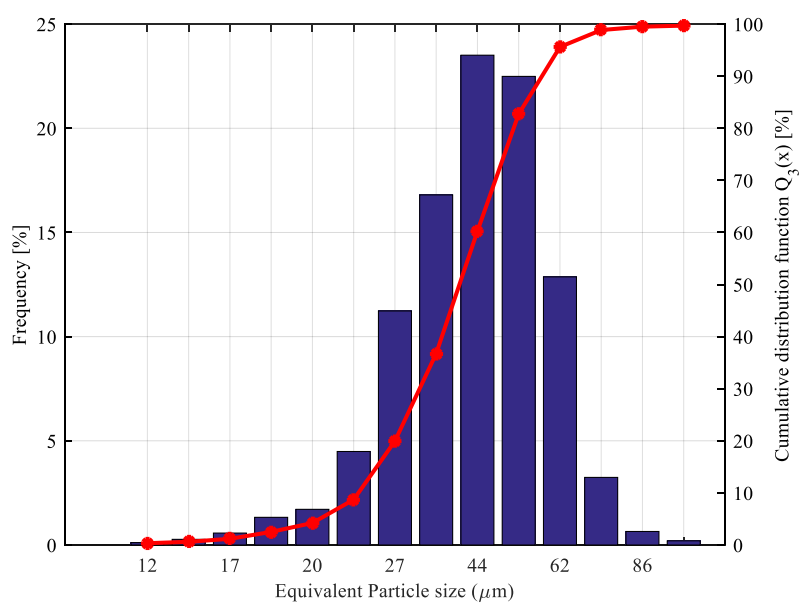

Figure 3. Particle size distribution of Akashiwo sanguinea used in the experiment: the cumulative volume fraction of algae cells below $62 \mu \mathrm{m}$ is close to $95 \%$
Akashiwo sanguinea is $36 \mu \mathrm{m}-52 \mu \mathrm{m}$ wide and $45 \mu \mathrm{m}-75$ $\mu \mathrm{m}$ long. A LIST-100 B-type field laser particle size meter is used for statistical observation of the samples of Akashiwo sanguinea. The particle size characteristics are as shown in Figure 3. The main particle size distribution is concentrated between $27 \mu \mathrm{m}$ and $62 \mu \mathrm{m}$ and in the form of a monomodal distribution. The equivalent particle diameter $D_{50}$ is about 40 $\mu \mathrm{m}$. In the later stage of exponential growth, the algae liquid is mixed and shaked, and then the solution of 17 kinds of concentration used in the experiment is arranged in the clean seawater sample. The number of algae cells is $0.13 \times$ $10^{5} \sim 5.02 \times 10^{5}$ cells/L.

\subsection{Design of platform for experimental facility}

To prevent the side wall effect, the ADV (Nortek AS) test is carried out in a $100 \mathrm{~L}$ vat fixed vertically to the water. Before each measurement, it is necessary to stir the algal fluid so that the cells can be evenly distributed. The ADV is controlled by the software of the computer to set parameters and turn on and off the instrument. The measurement data are recorded and stored in real time, and then analyzed on the computer through other analysis programs.

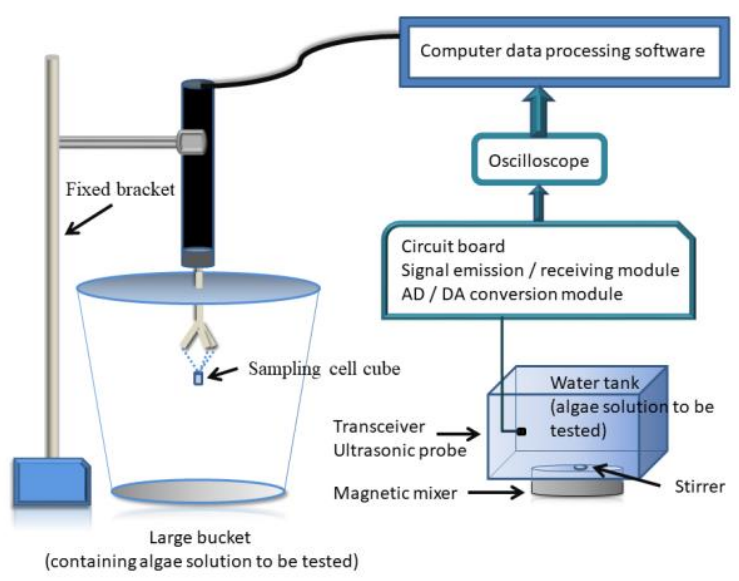

Figure 4. System experiment block diagram (left side) ADV actual measurement system (right side) transmitting and receiving ultrasonic probe and DB system

$\mathrm{DB}$ of single transmitting and receiving ultrasonic probe is used in the water tank $(300 \times 200 \times 147 \mathrm{~mm})$. The probe is suspended in the center of the water tank and placed horizontally. A thermometer is suspended on the left side wall to monitor the solution temperature during the experimental operation. The magnetic stirrer is placed in the middle of the bottom of the water tank. In order to ensure the uniform mixing of the algae liquid, the signal is collected after starting for 30 seconds and standing for a period of time for each 10 times. The other end of the probe is connected to the programmable circuit board system, and the system board controls the triggering and receiving switching of the ultrasonic signal. In the transmitting state, the electric signal is converted into the acoustic signal through the DA conversion module, and the acoustic signal acts on different concentrations of Akashiwo sanguinea in the water tank through the probe, and generates the corresponding backscatter signals that are received by the probe. When switching to the receiving mode, the received acoustic signal is converted to an electrical signal through the $\mathrm{AD}$ conversion 
module. After on-board filtering (amplification), real-time display and sampling are conducted by digital oscilloscope UT2102CE (sampling frequency up to $1 \mathrm{GS} / \mathrm{s}$ ). Finally, the collected data signals are analyzed and processed by the PC terminal, and the analysis results of the acoustic characteristics of different concentrations of algae solution are obtained. The probe excitation square-wave pulse frequency is $1 \mathrm{Mhz}$ and sends once every 30s (avoid the interference superposition of the signal). Each concentration group test record repeats 80 times and the algae solution of the same concentration is tested separately in 2 parallel samples

The ultrasonic probe has an outer diameter of $8 \mathrm{~mm}$, a nominal center frequency of $1 \mathrm{Mhz}( \pm 30 \mathrm{Khz})$, a half power angle of $6^{\circ}$ and a sharp angle of $14^{\circ}$, respectively. D is the diameter of the probe disc, and the far-field distance is calculated by Formula (6), and the far-field condition is satisfied.

$\mathrm{N}=\frac{D^{2}-\lambda^{2}}{4 \lambda}$

\section{EXPERIMENTAL RESULT AND DATA ANALYSIS}

\subsection{Basic characteristics of ADV test signal}

The ADV's probe is designed as a claw type with a threedimensional angle of $120^{\circ}$. To avoid interference factors such as interface reflection (Figure 5, bottom reflection signal of a certain measurement indicated by $350 \mathrm{~mm}$ in the upper right frame), the experiment is carried out in a $100 \mathrm{~L}$ white vat, so only multiple measurements are made for the low concentration of Akashiwo sanguinea (sufficient to verify the validity of the experiment). When the real-time recorded signal is processed, sampling data after 10 minutes of the same operation is taken, and abnormal groups are eliminated. And the waveforms of the three ultrasonic detection rod signals are slightly different, so the average value of the set after multitest of 3 rods is taken for characterization $(1$ count $=0.45 \mathrm{~dB})$.

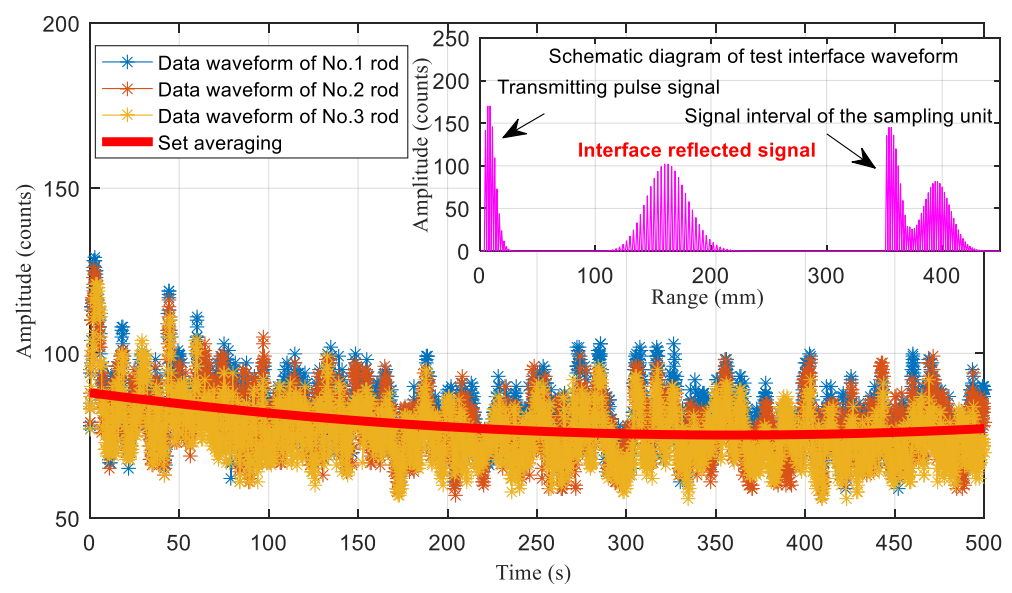

Figure 5. Basic test signal of ADV (1) the upper right box is the ADV software observation window signal (2) actual acquisition signal waveform

\subsection{Basic characteristics of backward acoustic scattered signal received by $D B$ probe}

When plane wave is incident, the amplitude of stationary random signal in the sound range is $V_{i}$ whose mean square value $E_{v}$ is consistent with sound pressure and proportional to sound strength. $D_{1}$ and $D_{0}$ correspond to the initial and end points of sampling, respectively. At low concentration, $E_{v}$ value is taken as the effective voltage value of the algae solution of this concentration. The ratio of $E_{v}$ value to the reference value measured by pure seawater gives the corresponding IBS value.

$E_{v}=\frac{1}{D_{1}-D_{0}} \sum V_{i}^{2}$

When the concentration of alga solution increases gradually, the envelope of the backscattered signal will attenuation exponentially (Figure 6 (a), $p_{0}$ is transmission sound pressure, $p$ is sound pressure at the distance $d$, and $\alpha$ is attenuation coefficient during propagation.

$p=p_{0} \mathrm{e}^{-\alpha d}$
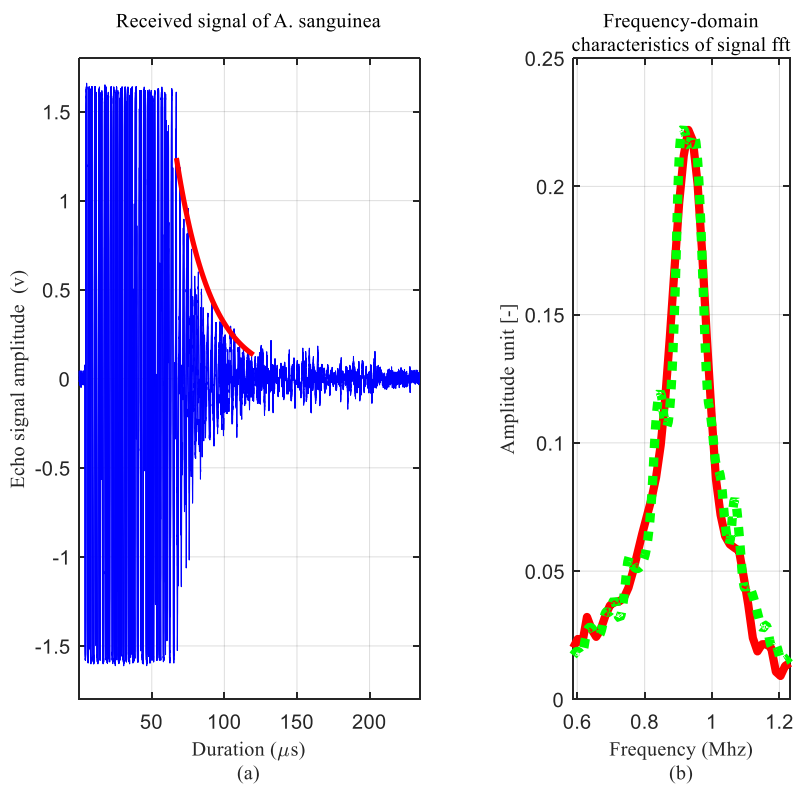

Figure 6. Envelope and frequency spectrum of measured high concentration signal (a) basic characteristics of received signal (b) frequency spectrum 
In addition to the characteristic of the ultrasonic probe, the attenuation of the signal is related to the attenuation due to the action of the algae cells and the absorption of the seawater in the Akashiwo sanguinea solution during the transmission of the ultrasonic wave. In (b), the peak of the frequency spectrum is at about $0.94 \mathrm{Mhzw}$ which slightly deviates from the probe frequency of $1 \mathrm{Mhz}( \pm 30 \mathrm{Khz})$, also indicating the presence of ultrasonic attenuation. On the premise that the far-field condition is satisfied, the signal within $30 \mu$ s after $70 \mu$ s from the initial point of the propagation signal is taken for analysis (indicated by the red full line in the detection area), and exponential curve fitting is performed to obtain the attenuation coefficient $a_{s}$ on the actual propagation path, where $c$ is the propagation velocity of the acoustic signal in the algal fluid, and $t$ is the propagation time.

$E_{v}=E_{0 v} \mathrm{e}^{-\alpha_{s} c t}$

\subsection{Comparison and analysis of basic experimental results}

The contrast acoustic measurements of the two ultrasound methods are carried out for 6 different kinds of concentration of Akashiwo sanguinea (to ensure that the concentration of the two is basically the same). The measurement result of ADV is transformed from count to $\mathrm{dB}$, and then the measured ratio of algae-containing solution to pure seawater solution is logarithmized. Although the performance (frequency, size) and system parameters of the probe used in the two methods are different, it can be seen from Figure 7 that the variation trend of their actual measurement with increasing concentration is basically consistent, and the linear relationship is good. In the low concentration range of $1 \times 10^{4}$ to $5 \times 10^{4}$ cells $/ \mathrm{L}$, when the number of per liter of algae cell is added by $1 \times 10^{4}$, the corresponding IBS is increased by about $0.6 \mathrm{~dB}$ ( $\mathrm{ADV}$ is $0.45 \mathrm{~dB}$ ). As the concentration increases, the increasing amplitude decreases slightly. The differentiation of ADV is good, and the linear correlation is obviously better than that of $\mathrm{DB}$, which is related to the performance advantage of the probe used in precision instruments. Therefore, to improve the performance of probe equipped on DB is one of the key factors for improvement of the whole platform system performance in the future.

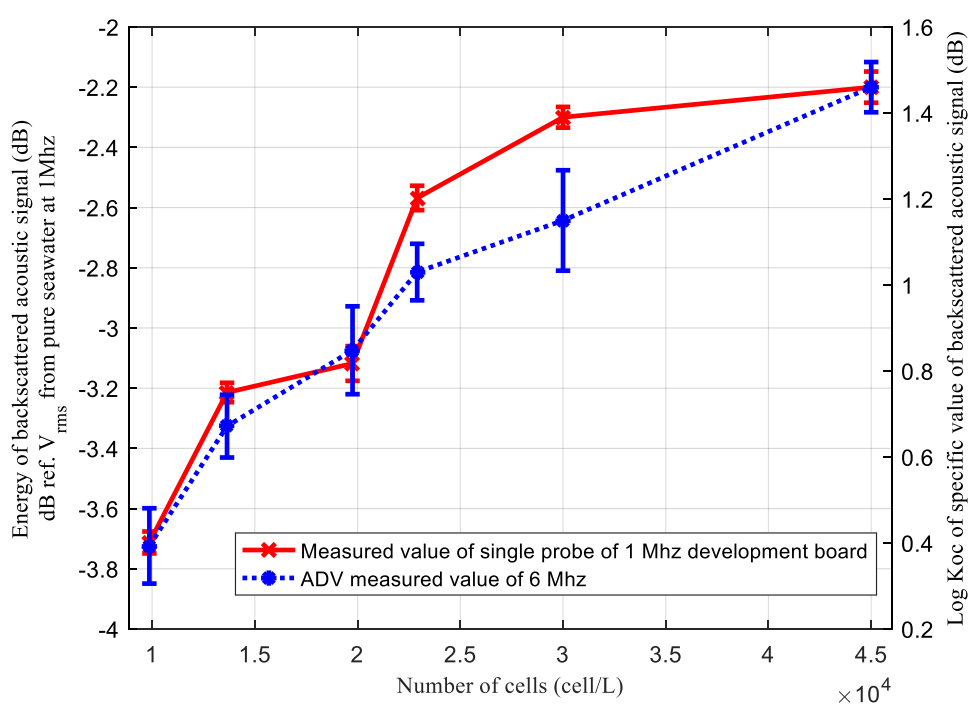

Figure 7. Comparison of acoustic test results of six konds of concentration (a) red full line, DB (1 Mhz probe) (b) blue dashed line, $\operatorname{ADV}(6 \mathrm{Mhz})$

\section{Discussion and Conclusions}

\subsection{Basic test situation and analysis of Mhz probe}

The ultrasonic backscattered signals of Akashiwo sanguinea collected by DB measurement system are filtered first, and then the time-domain statistical average processing and attenuation analysis are carried out in turn. It can be seen from Figure (a) that all the measured results are within the interval boundary (blue upper triangle, blue lower triangle full line) of the estimation range of the theoretical model calculated by formulas (4) and (5). The blue full line in the middle box corresponds to the estimated parameter result of Akashiwo sanguinea, and the measured results are in good agreement with the theoretical results under the estimated parameters.

The particle size of the cells is not a single value because the particle size is different in the microscopic examination of Akashiwo sanguinea. Therefore, assuming that mixing is uniform, the same distribution curve (Figure 3) is satisfied for all algal solution in the experiment. Therefore, under the theoretical estimation parameters of $g=1.05$ and $h=1.05$, the particle size of the theoretical value is corrected, as shown in Formula (10). Take 7 particle size distributions in the middle, calculate the individual characteristics of each particle size, and then combine to obtain the total backscattered strength value $S_{\mathrm{vN}_{\mathrm{M}}}$.

$\mathrm{S}_{\mathrm{vN}_{\mathrm{M}}}=10 \log _{10}\left(\sum_{\mathrm{i}=1}^{\mathrm{N}_{\mathrm{M}}} \mathrm{N}_{\mathrm{i}} \cdot \sigma_{\mathrm{i}}\right)+\mathrm{C}_{\mathrm{e}}$

From Figure 8 (b), it can be seen that the theoretical value after normalization is closer to that after the correction than before the particle size correction. Therefore, particle size correction is necessary and lays a foundation for the characterization of particle size in the course of microalgae acoustic detection in the later stage. 


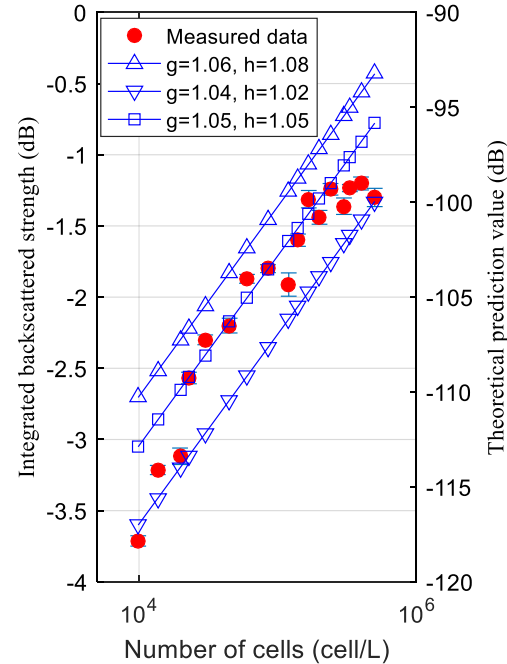

(a)

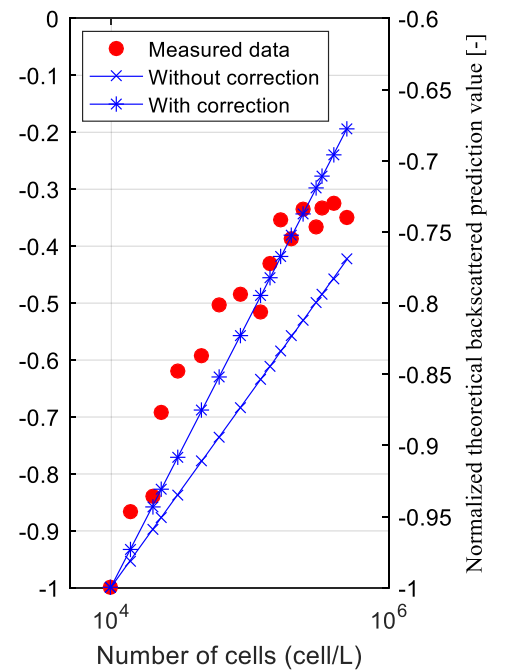

(b)

Figure 8. Comparison between the measured value and theoretical value (a) theoretical predicted value of different gh combination; (b) $\mathrm{g}=1.05, \mathrm{~h}=1.05$ comparison before and after the particle size correction

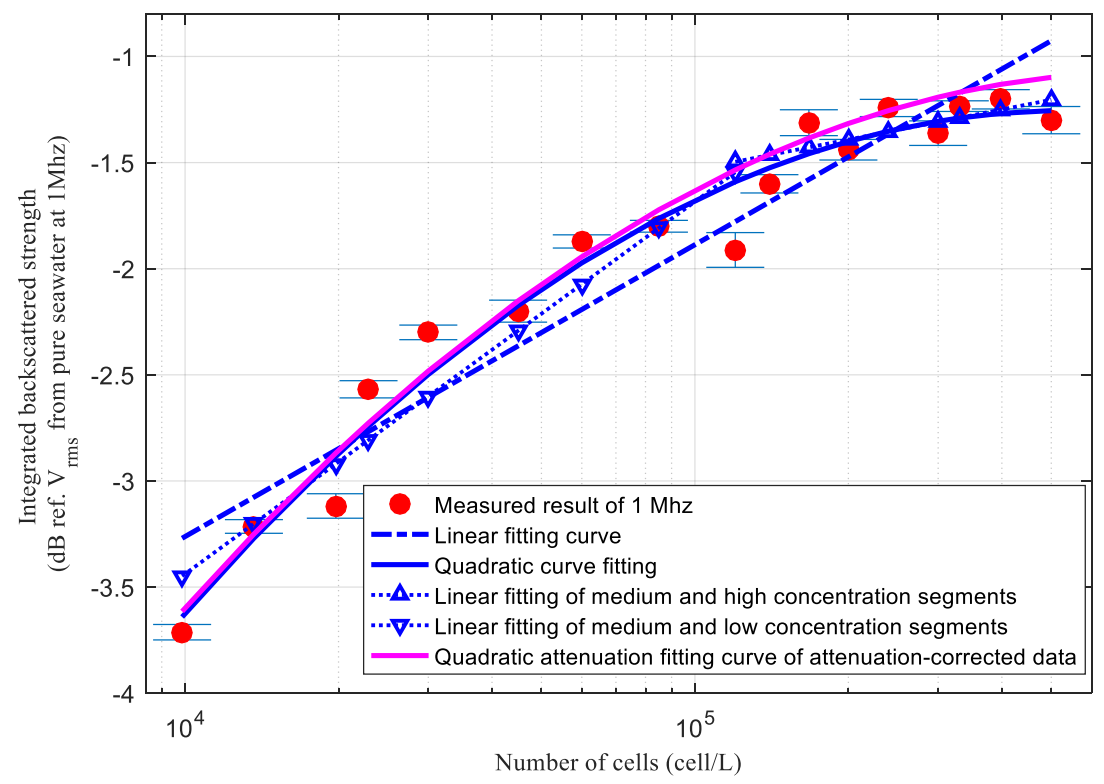

Figure 9. Analysis of backscattered acoustic signal of Akashiwo sanguinea by ultrasonic DB

In the comparison between theoretical and practical measurements, 17 acoustic measurements are fitted linearly. Although there is a significant correlation $\left(R^{2}=0.91\right)$, the increasing range of IBS value decreases with increasing concentration. The linear correlation trend of lowconcentration segment and high-concentration segment is different (Figure 9). The slope of low-concentration segment is obviously larger when they are segmented and fitted linearly again. After $2 \times 10^{5}$ cells/L, the fluctuation increases slowly because of the acoustic attenuation of the same ultrasonic emission signal by the high concentration algae solution. This is also illustrated by the time-frequency image of the received signal in Figure 6, so attenuation compensation is required. According to the principle of Formula (9), in the process of attenuation analysis of ultrasonic backscattered echo signal, it is found that the attenuation actually increases with the increase of algae cells in the solution. The points before correction are fitted with quadratic polynomial and the correlation coefficient is slightly increased, but the overall effect is more intuitionistic. The pink curve of Figure 9 is the fitting result after attenuation compensation. It can be seen that the amplitude at the medium and high concentration end $\left(>2 \times 10^{5}\right.$ cells $\left./ L\right)$ increases significantly compared with that before compensation. However, in the range of $0.1 \times$ $10^{5}$ cells/L to $0.9 \times 10^{5}$ cells/L, there is an increase but the attenuation is not obvious, and the correlation between IBS and algal cells is improved after compensation.

(Red dot) result of measured data (blue dashed line) linear fitting curve of measured data (blue full line) quadratic polynomial fitting of measured data (blue inverted triangle dashed line) linear fitting of medium and low concentration segment (blue positive triangle dashed line) linear fitting of medium and high concentration segment (pink full line) quadratic polynomial fitting curve after attenuation modification.

In the experiment proces, the measurement system has an uncertainty component which comes from the A type uncertainty $u_{A}$ measured repeatedly and from the imperfection 
of the system itself (measure method), such as uneven distribution of algae cells, B type uncertainty $u_{B}$ [31] for factors such as electrical noise during operation of the instrument. The former is obtained by statistical averaging of independent multiple measurements of the algal fluid samples, and the standard deviation of the IBP average is $S D_{n}(\bar{\alpha})$, which is calculated according to the Formula (10).

$u_{A}=S D_{n}(\bar{\alpha})=\sqrt[2]{\frac{1}{n(n-1)} \sum_{i=1}^{n}\left(\alpha_{i}-\bar{\alpha}\right)^{2}}$

The B type uncertainty is evaluated by theoretical and empirical analysis, and the combined uncertainty $U$ can be represented by formulas (11) and (12), respectively, where $\mathrm{k}=$ 2.

$u_{B}=\frac{1}{2} \sqrt{u_{B t}^{2}+u_{B v}^{2}}$
$U=k \sqrt{u_{A}^{2}+u_{B}^{2}}$

The same circuit system is used in the experiment and the ultrasonic probe is the same. We assume that the position of the fixed probe is not changed, the magnetic stirring is equivalent to the static treatment operation in the test, and the algae solution to be detected is uniformly mixed and then configured. Therefore, the test errors caused by the liquid temperature (error $\pm 0.5^{\circ} \mathrm{C}$ ) and volume (error $\pm 1 \%$ ) of B type uncertainty are mainly discussed here. ARD/V and ARD/t represent volume change, the average relative deviation of IBS caused by temperature change, respectively. $t^{+}, t^{-}, V^{+}$and $V^{-}$indicate the increase and decrease of temperature and increase and decrease of volume. By combining the calculated $u_{B}$ and $u_{A}$, the analysis result of the combined uncertainty $U$ can be obtained.

Table 1. Uncertainty in measurement of backscattered sound strength of DB for different concentration of Akashiwo sanguinea

\begin{tabular}{|c|c|c|c|c|c|c|c|c|}
\hline Item & \multicolumn{7}{|c|}{ Concentration / $\left(\times 10^{4}\right.$ cell $\left.\cdot L^{-1}\right)$} \\
\hline A- class uncertainty & 1.36 & 2.3 & 4.5 & 6.0 & 12.1 & 20.2 & 30.1 & 50.2 \\
\hline$u_{A}$ & $3.22 \%$ & $4.05 \%$ & $5.18 \%$ & $3.10 \%$ & $10.58 \%$ & $4.85 \%$ & $5.78 \%$ & $6.41 \%$ \\
\hline B- class uncertainty & 1.36 & 2.3 & 4.5 & 6.0 & 12.1 & 20.2 & 30.1 & 50.2 \\
\hline$u_{B}$ & $1.13 \%$ & $2.02 \%$ & $2.20 \%$ & $1.43 \%$ & $2.17 \%$ & $6.24 \%$ & $3.81 \%$ & $4.73 \%$ \\
\hline IBS $(\mathrm{dB})$ & -3.214 & -2.568 & -2.200 & -1.872 & -1.912 & -1.439 & -1.361 & -1.300 \\
\hline$t^{+}$ & -3.184 & -2.500 & -2.149 & -1.853 & -1.884 & -1.329 & -1.293 & -1.223 \\
\hline$t^{-}$ & -3.275 & -2.545 & -2.271 & -1.922 & -1.978 & -1.581 & -1.434 & -1.396 \\
\hline $\mathrm{ARD} / t$ & $1.57 \%$ & $2.88 \%$ & $2.92 \%$ & $2.11 \%$ & $2.77 \%$ & $9.06 \%$ & $5.23 \%$ & $6.86 \%$ \\
\hline$V^{+}$ & -3.277 & -2.645 & -2.280 & -1.916 & -1.986 & -1.571 & -1.448 & -1.389 \\
\hline$V^{-}$ & -3.182 & -2.504 & -2.141 & -1.851 & -1.867 & -1.331 & -1.307 & -1.223 \\
\hline $\mathrm{ARD} / V$ & $1.63 \%$ & $2.82 \%$ & $3.31 \%$ & $1.93 \%$ & $3.35 \%$ & $8.57 \%$ & $5.54 \%$ & $6.52 \%$ \\
\hline Synthesis uncertainty & 1.36 & 2.3 & 4.5 & 6.0 & 12.1 & 20.2 & 30.1 & 50.2 \\
\hline$U$ & $6.83 \%$ & $9.05 \%$ & $11.26 \%$ & $6.84 \%$ & $21.61 \%$ & $15.80 \%$ & $13.84 \%$ & $15.94 \%$ \\
\hline
\end{tabular}

Table 1 shows the results of uncertainty analysis of backscattered strength of Akashiwo sanguinea solution of different concentrations by ultrasonic excitation of $1 \mathrm{Mhz}$ in DB. In alga solution with concentration of $1.2 \times 10^{5} \mathrm{cell} / \mathrm{L}$, the relative measurement uncertainty component of A type is large, and the maximum relative measurement uncertainty of the system is $21.61 \%$, The results are in good agreement with the results in Figure 9, but they are still within the reasonable range of application of experimental measurement.

\subsection{Conclusions}

By using two sets of acoustic systems to measure and analyze the ultrasonic backscattered signal of 17 kinds of concentrations of Akashiwo sanguinea, the results show that in the pure sea algae environment, when the concentration reaches the minimum required range, $\mathrm{ADV}$ is able to detect microalgae and the detection performance is good. In the range of $0.1 \times 10^{5}$ to $0.5 \times 10^{5}$ cells $/ \mathrm{L}$, the trend of using $\mathrm{DB}$ is basically the same as the measurement result of ADV, and the IBS value increases with the increase of the concentration. The measured value of DB testing system is basically in accordance with the trend of the theoretical prediction value. In particular, the theoretical value after the particle size correction provides the basis and possibility for the subsequent feedback of the particle size characteristics. The attenuation of absorption and scattering caused by the increase of algae concentration is gradually obvious, and the statistical analysis of the envelope of the received signal also proves this phenomenon. The attenuation at low concentration is very small. At high concentration, the inhibition and attenuation of alga cells to the sound propagation process has obvious influence on the detection results, so it is necessary to compensate the attenuation. In the range of $0.1 \times 10^{5} \mathrm{cell} / \mathrm{L}$ to $5 \times 10^{5} \mathrm{cell} / \mathrm{L}$, there is a good linear correlation between the concentration and backscattered strength of Akashiwo sanguinea, which can meet the application range and requirement of red tide forecast. Taking $0.9 \times 10^{5} \mathrm{cell} / \mathrm{L}$ to $2.0 \times 10^{5} \mathrm{cell} / \mathrm{L}$ as the boundary segment, the linear change rate of medium and low concentration is significantly higher than that of medium and high concentration. The high concentration segment shows linear fluctuation increase, and the linear correlation of the system is enhanced after compensation.

To sum up, although there are uncertain factors in the experimental process, the whole measurement process is in a reasonable range, and the results can be appied to the monitoring of other algae species. With the combination of curve fitting and piecewise linear fitting, the use of single frequency and single probe is extended to the combination of multiple frequency and multiple probe, which is the future research direction of improving the system precision and diversifying the performance. The results of this study can provide some references for the observation and monitoring of red tide in China, and have significant scientific and practical significance, and are expected to be widely used in the prediction, monitoring and prevention of marine red tide. 


\section{REFERENCES}

[1] Smayda TJ. (1997). Harmful algal blooms: Their ecophysiology and general relevance to phytoplankton blooms in the sea. Limnology and Oceanography 42(5part2):

$1137-1153$

https://doi.org/10.4319/1o.1997.42.5_part_2.1137

[2] Landsberg JH. (2002). The effects of harmful algal blooms on aquatic organisms. Reviews in Fisheries Science 10(2): 113-390. https://doi.org/10.1080/20026491051695

[3] Hallegraeff GM. (2010). Ocean climate change, phytoplankton community responses, and harmful algal blooms: A formidable predictive challenge1. Journal of Phycology 46(2): 220-235. https://doi.org/10.1111/j.1529-8817.2010.00815.x

[4] Anderson DM, Andersen P, Bricelj VM. (2001). Monitoring and management strategies for harmful algal blooms in coastal waters. Unesco.

[5] Beardall J, Stojkovic S. (2006). Microalgae under global environmental change: Implications for growth and productivity, populations and trophic flow. ScienceAsia 32(S1): 001. https://doi.org/10.2306/scienceasia15131874.2006.32(s1).001

[6] Ebenezer V, Lim WA, Ki JS. (2014). Effects of the algicides $\mathrm{CuSO} 4$ and $\mathrm{NaOCl}$ on various physiological parameters in the harmful dinoflagellate Cochlodinium polykrikoides. Journal of Applied Phycology 26(6): 2357-2365. https://doi.org/10.1007/s10811-014-0267-9

[7] Lee TJ, Nakano K, Matsumara M. (2001). Ultrasonic irradiation for blue-green algae bloom control. Environmental Technology 22(4): 383-390. https://doi.org/10.1080/09593332208618270

[8] Ahn YH, Shanmugam P, Chang KI, Moon JE, Ryu JH. (2005). Spatial and temporal aspects of phytoplankton blooms in complex ecosystems off the Korean coast from satellite ocean color observations. Ocean Science Journal 40(2): 67-71. https://doi.org/10.1007/bf03028587

[9] Stumpf RP. (2001). Applications of satellite ocean color sensors for monitoring and predicting harmful algal blooms. Human and Ecological Risk Assessment: An International Journal 7(5): 1363-1368. https://doi.org/10.1080/20018091095050

[10] Stanton TK. (1988). Sound scattering by cylinders of finite length. I. Fluid cylinders. The Journal of the Acoustical Society of America, 83(1): 55-63. https://doi.org/10.1121/1.396184

[11] Stanton, T. (1996). Acoustic scattering characteristics of several zooplankton groups. ICES Journal of Marine Science 53(2): 289-295. https://doi.org/10.1006/jmsc.1996.0037

[12] Frisk GV, Hays EE. (1978). Acoustical oceanography, principles and applications. Deep Sea Research 25(8): 749. https://doi.org/10.1016/0146-6291(78)90629-x

[13] Holliday DV, Pieper RE. (1980). Volume scattering strengths and zooplankton distributions at acoustic frequencies between 0.5 and $3 \mathrm{MHz}$. The Journal of the Acoustical Society of America 67(1): 135-146. https://doi.org/10.1121/1.384472

[14] Kristensen $\AA$, Dalen J. (1986). Acoustic estimation of size distribution and abundance of zooplankton. The Journal of the Acoustical Society of America 80(2): 601611. https://doi.org/10.1121/1.394055
[15] Blanc S, Mosto P, Benitez C, Jua'rez R, Milou M, Lascalea G. (1998). Acoustical response of phytoplankton volume scatterers at ultrasonic frequencies as an indicator of pollution in sea waters. Transactions on Ecology and the Environment 18: 231 240. https://doi.org/10.2495/CENV980221

[16] Bok TH, Na J, Paeng DG. (2013). Diel variation in highfrequency acoustic backscatter from Cochlodinium polykrikoides. The Journal of the Acoustical Society of America 134(2): EL140-EL146. https://doi.org/10.1121/1.4812437

[17] Kim J, Choi JW, Kang D. (2012). Laboratory experiment to measure 5-MHz volume backscattering strengths from Red-tide causing microalgae Chattonella antiqua. Ocean Science Journal 47(3): 173-179. https://doi.org/10.1007/s12601-012-0017-3

[18] Bok TH, Paeng DG, Kim E, Na J, Kang D. (2010). Ultrasound backscattered power from Cochlodinium polykrikoides, the main red tide species in the Southern Sea of Korea. Journal of Plankton Research 32(4): $503-$ 514. https://doi.org/10.1093/plankt/fbq001

[19] Poindexter CM, Rusello PJ, Variano EA. (2010). Acoustic doppler velocimeter-induced acoustic streaming and its implications for measurement. Experiments in Fluids 50(5): 1429-1442. https://doi.org/10.1007/s00348-010-1001-2

[20] Hosseini SA, Shamsai A, Ataie-Ashtiani B. (2006). Synchronous measurements of the velocity and concentration in low density turbidity currents using an Acoustic Doppler Velocimeter. Flow Measurement and Instrumentation 17(1): 59-68. https://doi.org/10.1016/j.flowmeasinst.2005.05.002

[21] Fugate DC, Friedrichs CT. (2002). Determining concentration and fall velocity of estuarine particle populations using ADV, OBS and LISST. Continental Shelf Research 22(11-13): 1867-1886. https://doi.org/10.1016/s0278-4343(02)00043-2

[22] Hay AE. (1983). On the remote acoustic detection of suspended sediment at long wavelengths. Journal of Geophysical Research 88(C12): 7525. https://doi.org/10.1029/jc088ic12p07525

[23] Morse PM. (1948). Vibration and Sound. Second edition, 468 pages, illustrations, $15 \times 23 \mathrm{~cm}$. New York, McGraw-Hill Book Co., Inc.

[24] Hinton R, Dobrota M. (1976). Density gradient centrifugation. Laboratory Techniques in Biochemistry and Molecular Biology. Vol. 6, part 1. Edited by T. S. Work and E. Work. North-Holland Publishing Company, Amsterdam, 290.

[25] Peeters H. (1979). Separation of cells and subcellular elements. Pergamon Press, Oxford, 116.

[26] Chu D, Wiebe P, Copley N. (2000). Inference of material properties of zooplankton from acoustic and resistivity measurements. ICES Journal of Marine Science 57(4): 1128-1142. https://doi.org/10.1006/jmsc.2000.0800

[27] Eppley RW, Holmes RW, Strickland JDH. (1967). Sinking rates of marine phytoplankton measured with a fluorometer. Journal of Experimental Marine Biology and Ecology 1(2): 191-208. https://doi.org/10.1016/0022-0981(67)90014-7

[28] Oliver RL, Kinnear AJ, Ganf GG. (1981). Measurements of cell density of three freshwater phytoplankters by density gradient centrifugation. Limnol Oceanogr 26(2): 285-294. https://doi.org/10.4319/1o.1981.26.2.0285 
[29] Van Ierland ET, Peperzak L. (1984). Separation of marine seston and density determination of marine diatoms by density gradient centrifugation. Journal of Plankton Research 6(1): 29-44. https://doi.org/10.1093/plankt/6.1.29

[30] Guillard RRL, Ryther JH. (1962). Studies of marine planktonic diatoms.I. Cyclotella nana (Hustedt) and
Detonula confervacea (Cleve) Gran. Canadian Journal of Microbiology 8(2):

https://doi.org/10.1139/m62-029

[31] Gluschke M, Wellmitz J, Lepom P. (2004). A case study in the practical estimation of measurement uncertainty. Accreditation and Quality Assurance 10(3): 107-111. https://doi.org/10.1007/s00769-004-0895-x 